\title{
An Algorithm to Estimate Delay Times in Petri Net Models of Signaling Pathways with Experimental Data (Invited Paper)
}

\author{
Kanji Hioka \\ Graduate School of Science \\ and Engineering, Yamaguchi \\ University \\ 1677-1 Yoshida, Yamaguchi \\ 753-8512, Japan \\ hioka@ib.sci.yamaguchi- \\ u.ac.jp \\ Qi-Wei Ge \\ Faculty of Education, \\ Yamaguchi University \\ 1677-1 Yoshida, Yamaguchi \\ 753-8513, Japan \\ gqw@yamaguchi-u.ac.jp
}

\author{
Yoshimasa Miwa \\ Graduate School of Science \\ and Engineering, Yamaguchi \\ University \\ 1677-1 Yoshida, Yamaguchi \\ 753-8512, Japan \\ miwa@ib.sci.yamaguchi- \\ u.ac.jp \\ Hiroshi Matsuno \\ Graduate School of Science \\ and Engineering, Yamaguchi \\ University \\ 1677-1 Yoshida, Yamaguchi \\ 753-8512, Japan \\ matsuno@sci.yamaguchi- \\ u.ac.jp
}

\author{
Chen Li \\ Human Genome Center, \\ University of Tokyo \\ 4-6-1 Shirokanedai, Minatoku, \\ Tokyo 108-8639, Japan \\ chenli@ims.u-tokyo.ac.jp \\ Satoru Miyano \\ Human Genome Center, \\ University of Tokyo \\ 4-6-1 Shirokanedai, Minatoku, \\ Tokyo 108-8639, Japan \\ miyano@ims.u- \\ tokyo.ac.jp
}

\begin{abstract}
In this paper, we propose an algorithm to estimate delay times in a Petri net model of signaling pathways based on biological experimental data. Firstly, we demonstrate a modeling method of signaling pathways with discrete Petri net using ErbB4 signaling pathway. Then, we propose a delay time estimation algorithm for Petri net models with experimental data. The estimated delay times are assigned to the transitions of the Petri net model of the ErbB4 signaling pathway that is simulated on Cell Illustrator 3.0. The simulation results are evaluated by comparing with the experimental data.
\end{abstract}

\section{Keywords}

Signaling pathway, Petri nets, Model decomposition, Delay time estimation

\section{INTRODUCTION}

Signaling pathways regulate elaborate cell communication mechanisms by controlling various alteration procedures of cell behavior, such as cell growth, survival, proliferation, and apoptosis. Under such cellular communication mechanism, cell activities could be preciously governed and maintained in a good condition along with other biochemical interactions and processes.

Permission to make digital or hard copies of all or part of this work for personal or classroom use is granted without fee provided that copies are not made or distributed for profit or commercial advantage and that copies bear this notice and the full citation on the first page. To copy otherwise, to republish, to post on servers or to redistribute to lists, requires prior specific permission and/or a fee.

Bionetics '08, November 25-28, 2008, Hyogo, Japan.

Copyright 2008 ICST 978-963-9799-35-6
Recently, Li et al. [1] proposed a qualitative modeling method by paying attention to the molecular interactions and mechanisms using discrete Petri nets. Further, they proposed a timed Petri net based method of determining the delay times of transitions, i.e., the time taken in firing each transition. Their basic consideration is that the number of tokens flowed into a place is equivalent to the number of tokens flowed out. They also performed computational simulations of apoptosis as a running example along with obtained delay times [2]. However, the estimation of a delay time in their proposal did not consider biological experimental data. Therefore, in order to carry out more precise simulation that could reproduce the facts described in biological experiments and/or scientific literature, we propose a new method to construct Petri net model of a signaling pathway that estimates a delay time based on given experimental data.

In this paper, we first model signaling pathways with discrete Petri net using the modeling method proposed by Li et al. [1]. Then, we propose a delay time estimation algorithm for a Petri net model with provided concentration behaviors of proteins depicted by Hatakeyama et al. [3] that are regarded as biological experimental data. Finally, the availability of proposed algorithm is confirmed through computational experiments on ErbB4 receptor signaling pathways with a simulation tool Cell Illustrator 3.0..

\section{DEFINITIONS OF PETRI NETS}

Petri nets are powerful tools in modeling and simulating various concurrent systems [4], especially biological pathways because Petri nets have the following superior characteristics [5]:

(1) "firm mathematical foundation" enabling formal and clear description of biological pathways as well as their structural analysis, and

(2) "visual representation of networks" which provides intuitive understanding of biological pathways without any mathematical descriptions that are basically difficult for ordinary biol- 
ogists.

In this section, we only give the necessary definitions used in this paper.

\subsection{Petri Nets}

A Petri net $N$ is defined by a 5 -tuple $N=(T, P, E, \alpha, \beta)$ that corresponds to a bipartite graph, where $T$ is a set of transitions represented by bars or boxes, $P$ is a set of places represented by circles in a graph, $E$ is a set of directed arcs between places and transitions (Figure 1). Parameter $\alpha$ denotes the weight of an arc from place to transition, and parameter $\beta$ denotes the weight of an arc connected from transition to place.

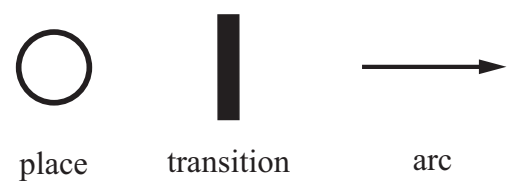

Figure 1: Basic elements of Petri net.

A place can hold a positive integer that represents the number of tokens. An assignment of tokens in places expressed in form of a vector is called marking $M$, which varies during execution of a Petri net. A transition without input places is called source transition that is always firable, and a transition without output places is called a sink transition.

[Firing rule of Petri net $N$ ] A transition $t$ is firable if each of input place $p_{I}$ of $N$ has at least $\alpha_{e}$ tokens, where $\alpha_{e}$ denotes the weight of an $\operatorname{arc} e=\left(p_{I}, t\right)$. Firing of a transition $t$ removes $\alpha_{e}\left(e=\left(p_{I}, t\right)\right)$ tokens from each input place $p_{I}$ of $t$ and deposit $\beta_{e}\left(e=\left(t, p_{O}\right)\right)$ tokens to each output place $p_{O}$ of $t$.

Figure 2 illustrates the movement of tokens by the firing of the transition. For the more details of Petri net, the readers are suggested to refer to Ref. [4].

\subsection{Timed Petri Nets}

With the extension of assigning delay time to each transition, Petri net becomes a powerful tool to observe the dynamic behavior of a system. Such extended Petri net is called timed Petri net $\bar{N}=(\bar{T}, \bar{P}, \bar{E}, \alpha, \beta)$. The firing rule of timed Petri net is defined as follows:

[Firing rule of timed Petri nets $\bar{N}$ ] (1) When the firing of a transition $t_{i}$ is decided, tokens required for the firing are reserved. (2)

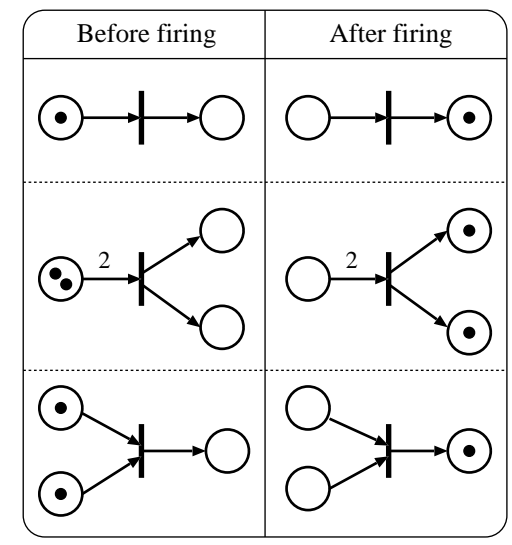

Figure 2: Examples of firing rules of Petri net.
After the delay time $d_{i}$ of transition $t_{i}, t_{i}$ fires to remove the reserved tokens from the input place of $t_{i}$ and put tokens into the output places of $t_{i}$.

In a timed Petri net, the number of firing per unit time is called firing frequency of a transition $t_{i}$. Accordingly, the firing frequency of a transition having the delay time $d_{i}$ can be expressed by $f_{i}$.

\section{MODELING OF SIGNALING PATHWAYS}

In this section, we give a modeling method with a set of modeling rules for signaling pathways based on Petri net representation. We use an example of ErbB4 signaling pathways to demonstrate our modeling method. Petri net based modeling of signaling pathways gives us intuitive understanding of the intrinsic structure and features of signaling pathways, and further enables computational experiments on the constructed Petri net model as being demonstrated in the following sections.

\subsection{Modeling Rules}

The structural characteristics of signaling pathways can be naturally and explicitly expressed by Petri net according to the following simple rules.

(1) Places denote static elements including chemical compounds, conditions, states, substances and cellular organelles participating in the biological pathways. Tokens indicate the presence of these elements. The number of tokens is given to represent the amount of chemical substances.

(2) Transitions denote active elements including chemical reactions, events, actions, conversions and catalyzed reactions.

(3) Directed arcs connecting the places and the transitions represent the relations between corresponding static elements and active elements. Arc weights $\alpha$ and $\beta$ describe the quantities of substances required before and after reaction, respectively. In case of modeling a chemical reaction, arc weights represent quantities given by stoichiometric equations of the reaction itself. Note that, weight of an arc is omitted if the weight is 1 .

Signaling pathways are information cascades of enzyme reactions from transmembrane receptors to the nucleus DNA, which ultimately regulate intracellular responses such as programmed cellular proliferation, gene expression, differentiation, secretion and apoptosis. For signaling pathways, besides the catalytic reactions, the information among the molecular interactions such as complex formation, gathering action, translocation and channel switching, need to be modeled according to different types of interactions as long as the biological facts that have been known.

Figure 3 shows various molecular interactions of signaling pathways and their correspondence to the Petri net models [1]. We give explanations about the molecular interactions appeared in the ErbB4 signaling pathway that is used in the next subsection to demonstrate our method. The pattern XIII in Figure 3 is newly added in this paper, which is necessary for the modeling of ErbB4 signaling pathway.

I. Association reaction is a binding reaction to induce the formation of homo- or heterodimers and to generate a complex compound. This block shows the ligand-receptor binding interaction. Obviously, we also can expand the concept of association to the formation of model represented in block $\mathbf{I}(\mathrm{b})$, generally representing the simultaneous association of substrates $S_{1}, \cdots, S_{n}(n \geq 1)$ forming a complex $C$ in a biological system.

II. Phosphorylation is a reaction to add a phosphate $\left(\mathrm{PO}_{4}\right)$ group 


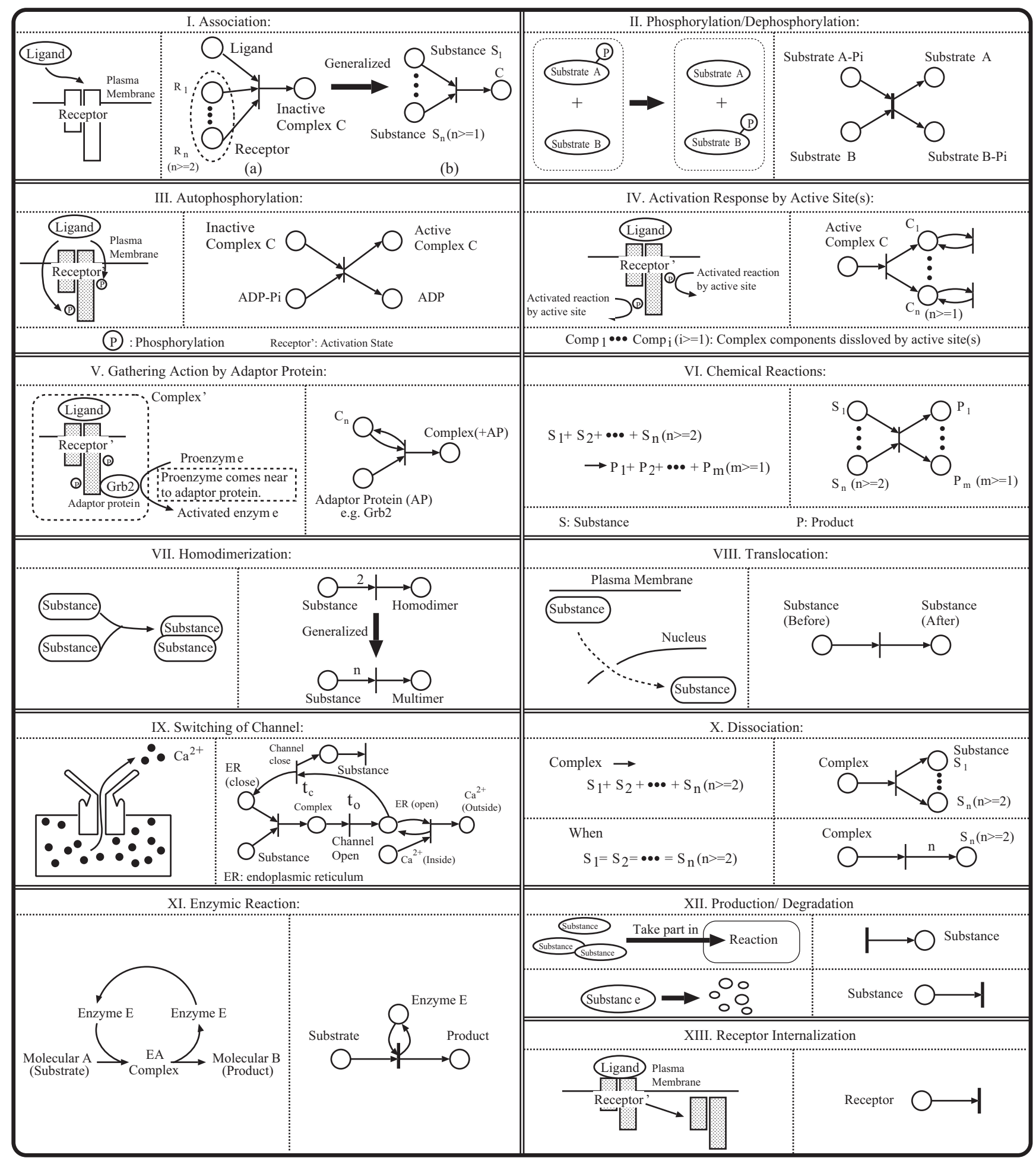

Figure 3: Petri net models of various reaction types in signaling pathways (Reaction types I to XII are presented in [1]).

to a protein or a small molecule, and dephosphorylation that is the backward reaction of phosphorylation removing phosphate groups from a compound by hydrolysis.

III. Autophosphorylation is a transphosphorylation reaction frequently following the binding of a ligand to a receptor with intrinsic protein kinase activity.

IV. Generally continually activated ligand-receptor complex regulates varied majority of cellular pathways transmitting the signals within the cell. Our basic consideration is that, if there are more than one successive signaling pathway that has distinct active 
site(s) (subunits) of activated complex, all the active site(s) shall be regarded as complex component(s) $C_{1}, \cdots, C_{n}(n \geq 1)$ as shown in block IV.

VII. Homodimerization is a polymerization reaction of two identical substances to form a dimmer similar to a kind of association reaction. A substance is modeled by an input place connected with a 2-weighted arc. It is easy to expand the conception to model the formation of multimer holding $n$-weight.

$\mathbf{X}$. This is the opposite reaction of $\mathbf{I}$. Dissociation process is a general process in which complexes and molecules separate or split into smaller molecules or ions. The number of input place of transitions is one while the output place number is two or more.

XI. Since an enzyme itself plays a role of catalyzer in biological pathways and there occurs no consumption in biochemical reactions, the reaction is modeled by a transition, whereas the substrate is modeled by enzyme place that has a self-loop with same arcweight.

XIII. Internalization is a phenomenon to decrease the number of receptors on the surface of a cell membrane, due to be exposed to corresponding biological agent such as ligands for a long time. As a result of decreasing the number of receptors, responsiveness of the ligands is decreased. The internalization is modeled by an output transition connected with a place denoting receptor via a normal arc.

\subsection{Modeling Example}

In this paper, we use the example of ErbB4 receptor signaling pathway to explain our method. ErbB tyrosine kinase receptors mediate mitogenic signal cascade by binding a variety of ligands such as EGF and recruiting the different cassettes of adaptor proteins. The binding of ligands to ErbB receptors results in diverse biological outputs, such as cellular proliferation and differentiation, and their deregulated expression or mutation highly correlates with the incidence of certain types of human cancer [3]. The ErbB4 signaling pathway is shown in Figure 4.

Figure 5 shows the whole Petri net model of ErbB4 receptor signaling pathways based on our modeling rules. Here we only explain a part of the modeling operation. Firstly, for the Receptor (R) -ligand (EGF) binding interaction, a Petri net model is obtained by applying the block $\mathbf{I}$ in Figure 4 in which two places $\left(p_{1}\right.$ and $\left.p_{2}\right)$ merge into a place $p_{3}$ that denotes R-EGF via transition $t_{1}$. Next, R-EGF forms a R-EGF2 dimer receptor to which we applied block VII the reaction model of homodimerization. Represented by transition $t_{2}$. Further, R-EGF2 conducts autophosphorylation to which we applied block III of the reaction model of autophosphorylation whose transition is labelled with transition $t_{3}$. Places $p_{5}, p_{6}$ and $p_{7}$ represent phosphorylated receptor RP, ATP and ADP, respectively. Transition $t_{7}$ represents the dephosphorylation (block III) of RP. $\mathrm{PI} 3 \mathrm{~K}\left(p_{33}\right)$ is activated by binding to the active sites $\left(p_{32}\right)$ which is modeled by the block IV. R-PI3K* $\left(p_{34}\right)$ is active PI3K. PR $\left(p_{35}\right)$ and $\mathrm{PI} \mathrm{K}^{*}\left(p_{36}\right)$ dissociate from $\mathrm{R}-\mathrm{PI}_{3} \mathrm{~K}^{*}\left(p_{34}\right)$, which is modeled by applying block $\mathbf{X}$ via the transition $t_{39}$. PI3K $\left(p_{33}\right)$ initiates the activation of $\mathrm{PI}\left(p_{38}\right)$. Since this reaction (represented by the transition $t_{44}$ ) does not consume PI3K, this can be modeled by using the enzyme place $\left(p_{36}\right)$ with a self-loop (see block XI). Transition $t_{42}$ represents the dephosphorylation (block III) of PI3K*. The remaining Petri net model in Figure 5 was constructed by applying the blocks in Figure 4 in the same way as described above.

\section{MODEL DECOMPOSITION}

Before the procedure of delay time estimation, we decompose a Petri net model into smaller components. The procedure of model decomposition is intended to independently determine a delay time

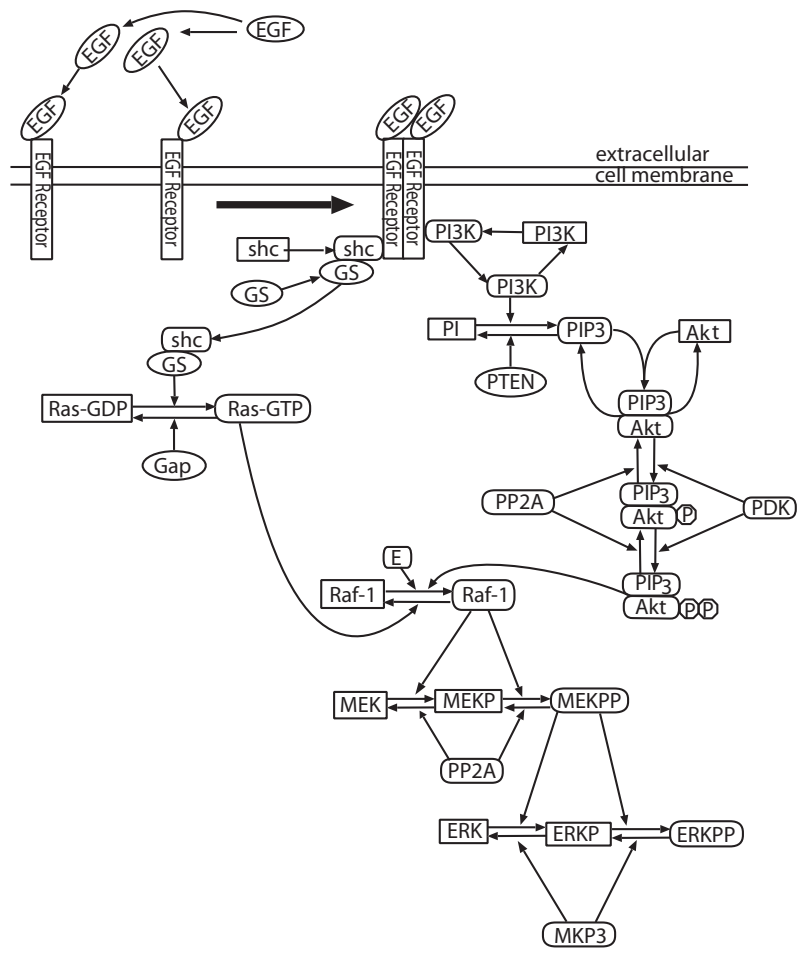

Figure 4: Biological diagram of ErbB4 signaling pathway.

within each component that is a subgraph of a Petri net model. This model decomposing procedure is designed based on the idea proposed in [6].

Note that, enzyme places possessing experimental data are called data places $d p_{n}(n \in N)$; for two data places $d p_{i}$ and $d p_{j}$ in a Petri net model, the condition $i<j$ implies that place $d p_{i}$ is located at upper position than place $d p_{j}$. When using timed Petri net to simulate signaling pathways, self-loops of enzyme places are replaced by test arcs with a threshold value.

A Petri net model is decomposed into components $C_{1}, C_{2}, \cdots, C_{r}$ $(r \leq 1)$ by choosing data place from upstream data places in order of increasing number and according to the following rules.

(i) A data place $d p_{n}$ to which an output transition is connected via a test arc, must exist in a component $C_{n}$.

(ii) For a place that exists only in a component $C_{n}$, all the transitions connected to this place via normal arcs must exit in a component $C_{n}$.

(iii) For a transition in a component $C_{n}$, all the places connected to this transition must be included in the same component $C_{n}$.

These properties are based on the assumptions that (a) the reactions denoted by these transitions determine the concentration levels of protein of places connected with a transition via normal arc(s), and (b) a transition connected with a place via a test arc does not affect the concentration level of the place.

To explain the method of decomposing a Petri net model, we use a simple example as shown in Figure 6. Firstly, we choose the most upstream data place $d p_{1}$ and find a component $C_{1}$ by conducting the above rules. According to the rule (i), place $d p_{1}$ is chosen as an element of component $C_{1}$ (block (1)). Thereafter, according to the rule (ii), the incoming and outgoing transitions of data place $d p_{1}$ are included in component $C_{1}$ (block (2)). Furthermore, according 


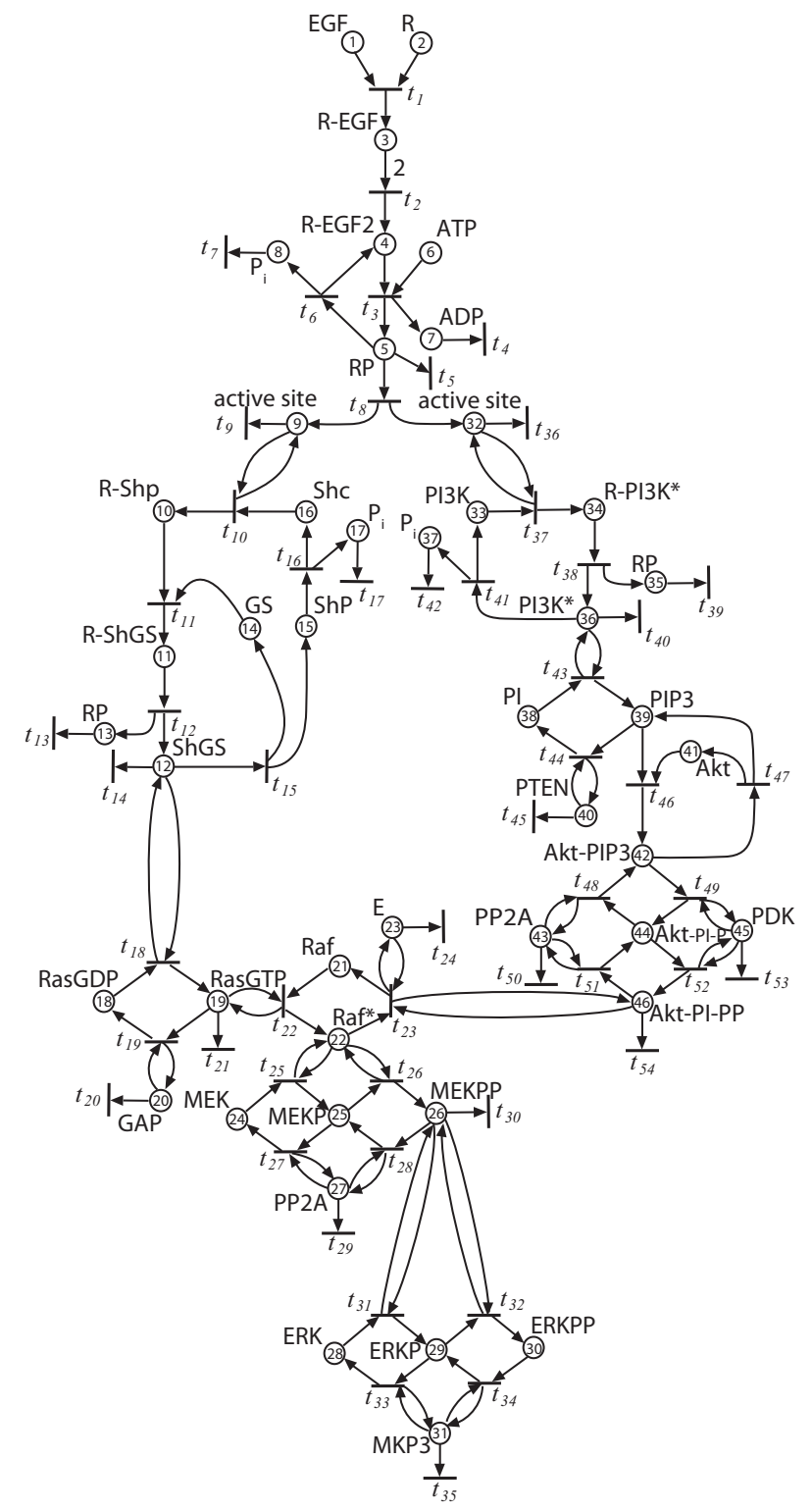

Figure 5: Petri net model of the ErbB4 signaling pathway.

to the rule (iii), the component $C_{1}$ includes all transitions that are attached to the places contained in the component $C_{1}$ (block (3)). By repeating the same actions as above, construction of component $C_{1}$ is completed. Then, we choose a next data place $d p_{2}$, and find component $C_{2}$ in the same way. Figure 7 shows the decomposed components of the Petri net model of Figure 5.

\section{ALGORITHM TO ESTIMATE DELAY TIME}

In our previous paper [2], we proposed an algorithm to determine delay times assigned to transitions in Petri net model of a signaling pathways. However, this algorithm does not employ existing biological data. This section therefore gives an improved algorithm that obtains delay times of transitions with considering biological data.

Signaling pathway is a signal transduction system that propagates signals from a receptor at the membrane to the nucleus. Hence,

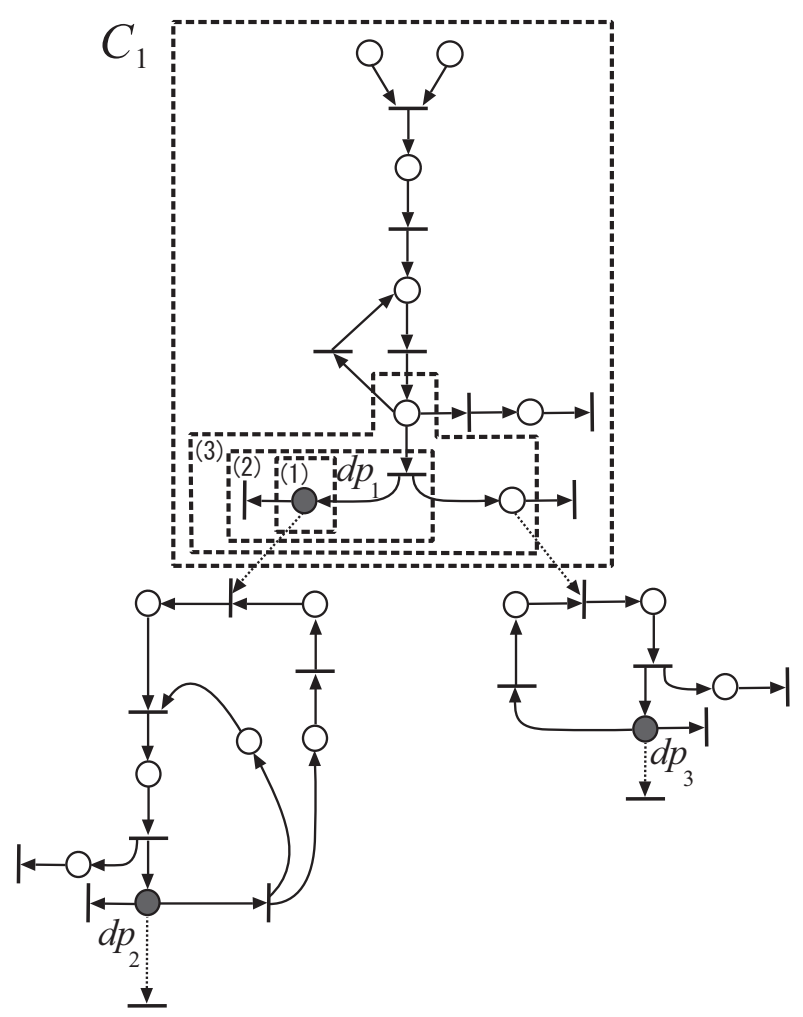

Figure 6: Example of model decomposition. Black colored places are data places $\left(d p_{n}\right)$.

we consider a time period from the activation of a receptor to the activation of a certain protein in a signaling pathway. In this paper, we use experimental data that are the graphs of concentration behaviors of activated enzymes in the ErbB4 signaling pathway presented in Ref.[3]. We used experimental data whose EGF concentration is at $10 \mathrm{nM}$ among several conditions of EGF concentration performed in Ref. [3]. Note that, when the activated enzyme concentration reaches the peak of concentration curve, the time point can be considered as the time point $D$, where an enzyme works most effectively after receiving extracellular stimuli. Table 1 summarizes the time points of peaks concerning to corresponding enzyme concentrations. These time points $D$ s are used for a delay time estimations.

Table 1: Experimental data extracted from Hatakeyama et al. [3].

\begin{tabular}{cc}
\hline Biological substance & Time point $D(\mathrm{sec})$ \\
\hline \hline RP & 22.9 \\
Raf $*$ & 36.9 \\
ShP & 69.9 \\
MEKPP & 192.7 \\
ERKPP & 643.1 \\
PI3K* & 73.3 \\
Akt-PI-PP & 232.1 \\
\hline
\end{tabular}

To conduct delay time estimation, we show the basic principle that is used construct a model which reproduces the biological events according to the biological data with the use of timed Petri 


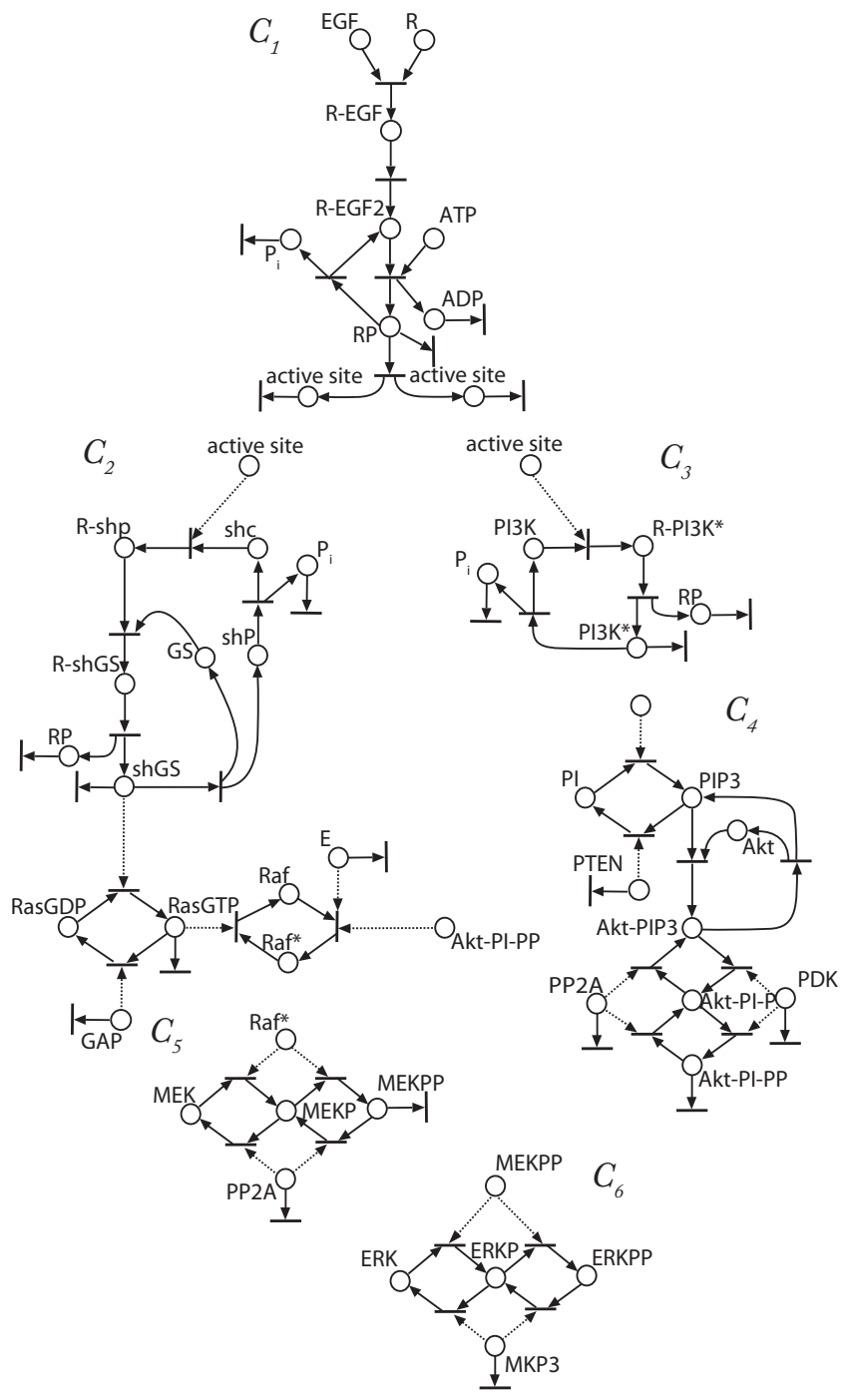

Figure 7: Components of a timed Petri net model of ErbB4 receptor signaling pathway. Self-loops are replaced by test arcs. Test arc is represented by dashed arc.

net model.

\section{[Basic principle]}

The sum of consumption should be the production in order to keep the concentration equilibrium for each substance engaged in signaling pathways, i.e. for each place the token amounts flowed in and flowed out per unit time should be equivalent:

$$
\sum_{i=1}^{m} K_{I_{i}}=\sum_{j=1}^{n} K_{O_{j}}
$$

$\sum_{i=1}^{m} K_{I_{i}}$ and $\sum_{j=1}^{n} K_{O_{j}}$ are the total token amounts flowed in and flowed out per unit time, respectively.

Because this sink transition is used to model slow speed degradation of an enzyme, the delay time $d_{i}$ of the sink transition is assigned with a large value as the minimum of the firing frequencies to the sink transitions. Here, the delay time $d_{i}$ of the sink transitions is assigned to 1000 due to the feature of small and natural degradation. Furthermore, in the case that a place has more than one output transitions, a so-called "conflict" occurred. That is, the token removal from the place by the firing of one output transition disables the firings of other output transitions. Therefore, this paper assumes such enabled transitions in conflict have the same chance to fire.

In the following, we will give our delay time estimation algorithm for Petri net model of signaling pathways. Notations and functions used in the algorithm are as follows:

(1) Let $P N_{c}=(P N, D C)$ denote a Petri net model that is decomposed according to the decomposition method stated in section 2, where $D C=\left\{C_{1}, C_{2}, \cdots, C_{k}\right\}$ and $C_{i} \in D C(i \in N)$ denotes each smaller component.

(2) Let $T_{u}$ denote a set of transitions that are not estimated.

(3) Let sizeof() be an operation that calculates the element number of the set.

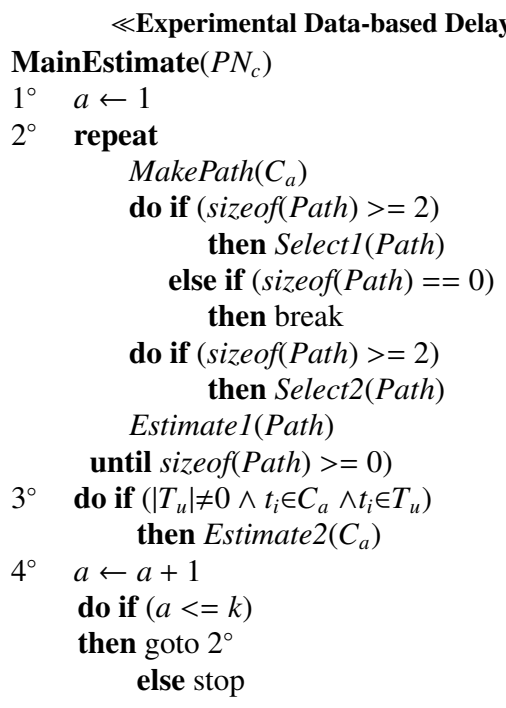

In the algorithm "Experimental Data-based Delay Time Estimation", the function of MakePath $\left(C_{a}\right)$ is designed to compute the shortest paths path $(i=1,2, \cdots)$ between any two data places in $C_{a}$, and Path $=\left\{\right.$ path $_{1}$, path $\left._{2}, \cdots\right\}$. It is satisfied that path $_{i} \in$ Path must include at least one transition without estimation in $C_{a}$. After that, one path $_{i} \in$ Path is selected to be estimated according to following cases:

(i) if $\mid$ Path $\mid \geq 2$ holds, the function of Selectl(Path) is invoked to compute and return the result of path ${ }_{i}$ that exists in the shortest path between most-upstream data place and mostdownstream data place in $C_{a}$;

(ii) if no element exists in Path, no operation is executed.

Then, if $\mid$ Path $\mid \geq 2$ holds, Select2(Path) is used to compute and return the result of path ${ }_{i} \in$ Path satisfying the condition that the number of transition of path $h_{i}$ is the least. In step $2^{\circ}$,Estimate 1(Path) is considered to compute the delay time $d_{(m, n), i}(i \in N)$ of un-estimated transitions in path $_{i}$ that is selected through the function of Select 1 or Select2. In the function of Estimate1(Path), two equations are used to calculate the delay time till the estimation of all the transitions in $\mathrm{path}_{i}$ finished.

$$
\begin{gathered}
d_{(m, n), 1}+d_{(m, n), 2}+\cdots+d_{(m, n), x}=D_{n}-D_{m} \\
\sum_{i=1}^{m} \frac{\beta_{i}}{d_{I_{i}}}=\sum_{j=1}^{m} \frac{\alpha_{j}}{d_{O_{j}}}
\end{gathered}
$$


Table 2: A delay time of transition in timed Petri net model (Figure 5).

\begin{tabular}{|c|c||c|c||c|c|}
\hline transition $\left(t_{i}\right)$ & delay time $\left(d_{i}\right)$ & transition $\left(t_{i}\right)$ & delay time $\left(d_{i}\right)$ & transition $\left(t_{i}\right)$ & delay time $\left(d_{i}\right)$ \\
\hline \hline$t_{1}$ & 2.5 & $t_{19}$ & 2.8 & $t_{37}$ & 25.2 \\
\hline$t_{2}$ & 4.9 & $t_{20}$ & 1000 & $t_{38}$ & 25.2 \\
\hline$t_{3}$ & 3.3 & $t_{21}$ & 1000 & $t_{39}$ & 1000 \\
\hline$t_{4}$ & 1000 & $t_{22}$ & 2.8 & $t_{40}$ & 1000 \\
\hline$t_{5}$ & 9.8 & $t_{23}$ & 2.8 & $t_{41}$ & 25.2 \\
\hline$t_{6}$ & 9.8 & $t_{24}$ & 1000 & $t_{42}$ & 1000 \\
\hline$t_{7}$ & 1000 & $t_{25}$ & 77.9 & $t_{43}$ & 39.7 \\
\hline$t_{8}$ & 9.8 & $t_{26}$ & 77.9 & $t_{44}$ & 39.7 \\
\hline$t_{9}$ & 1000 & $t_{27}$ & 77.9 & $t_{45}$ & 1000 \\
\hline$t_{10}$ & 2.8 & $t_{28}$ & 77.9 & $t_{46}$ & 39.7 \\
\hline$t_{11}$ & 2.8 & $t_{29}$ & 1000 & $t_{47}$ & 39.7 \\
\hline$t_{12}$ & 2.8 & $t_{30}$ & 1000 & $t_{48}$ & 39.7 \\
\hline$t_{13}$ & 1000 & $t_{31}$ & 225.2 & $t_{49}$ & 39.7 \\
\hline$t_{14}$ & 1000 & $t_{32}$ & 225.2 & $t_{50}$ & 1000 \\
\hline$t_{15}$ & 38.6 & $t_{33}$ & 225.2 & $t_{51}$ & 39.7 \\
\hline$t_{16}$ & 38.6 & $t_{34}$ & 225.2 & $t_{52}$ & 39.7 \\
\hline$t_{17}$ & 1000 & $t_{35}$ & 1000 & $t_{53}$ & 1000 \\
\hline$t_{18}$ & 2.8 & $t_{36}$ & 1000 & $t_{54}$ & 1000 \\
\hline
\end{tabular}

The left-hand member of (1) shows the sum of the delay time of all the transitions involved in path $_{i}$ between two selected data places. The right-hand member of (1) shows elapsed time between selected data places that they reach at the peak of the amount respectively, i.e., the difference between downstream data $\left(D_{n}\right)$ and upstream data $\left(D_{m}\right)$. Equation (2) is designed to calculate a delay time of transitions between back and forth places that exist between selected data places (Figure 8), and to obey Basic principle.

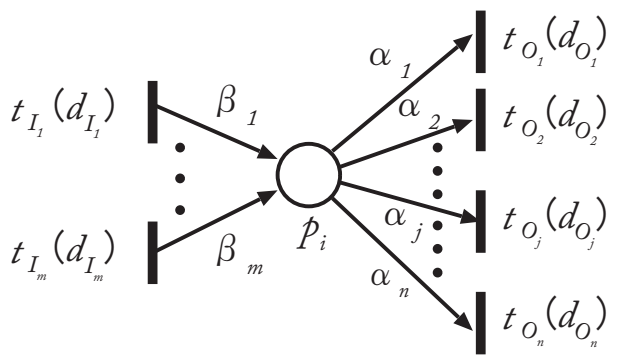

Figure 8: The illustration of equation (2). Place and transition are denoted as $p_{i}$ and $t_{i}$, respectively.

In step $3^{\circ}$, Estimate $2\left(C_{i}\right)$ is considered to compute the delay time of left transitions in $C_{i}$ without doing estimation. The delay time of such transition $t_{O_{j}}$ (see Figure 3 ) is obtained based on equation (2) in the context of its net structure.

Using this algorithm, a timed Petri net model with delay time of transitions can be constructed based on real experimental data. With this estimation, the performance variation along with time change may probably provide a number of valuable insights. Further, it can be considered that the simulation result is more precious to give new presumption and closed to the actual environment rather than the simple estimation according to topological structure proposed by Li et al. [2].

In the next section, we will do simulation to evaluate our estimation algorithm.

\section{SIMULATION OF SIGNAL FLOWS WITH CELL ILLUSTRATOR}

We applied the proposed algorithm for the Petri net model of ErbB4 signaling pathway in Figure 5. As experimental data, we used the simulation results given in Ref. [3] that are given in table 2. The obtained delay times in this Petri net model are shown in Table 2. This Petri net model was described with Cell Illustrator 3.0 (CI) [8] in which delay times of transitions in Table 2 were incorporated.

To simulate signal flows in the Petri net model, we need to assign the initial marking to the places that are responsible to token flows. We determined the number of minimal tokens so as to flow the tokens only once from most upstream place through most downstream place (Table 3 ). With the obtained delay times and the minimal initial markings, we observed the token flows on this model with CI. Figure 9 shows the token behaviors of places in Table 1. The times when the places get token exhibit almost the same as the time points $D$ in table 1 that are intervals from the ligand activation to the peak of biological substances.

Table 3: The number of initial tokens.

\begin{tabular}{|l|c|}
\hline Biological substance & Initial tokens \\
\hline \hline HRG & 2 \\
\hline R & 2 \\
\hline ATP & 1 \\
\hline Shc & 2 \\
\hline RasGDP & 2 \\
\hline Raf & 2 \\
\hline PI3K & 2 \\
\hline PI & 1 \\
\hline MEK & 2 \\
\hline ERK & 1 \\
\hline
\end{tabular}



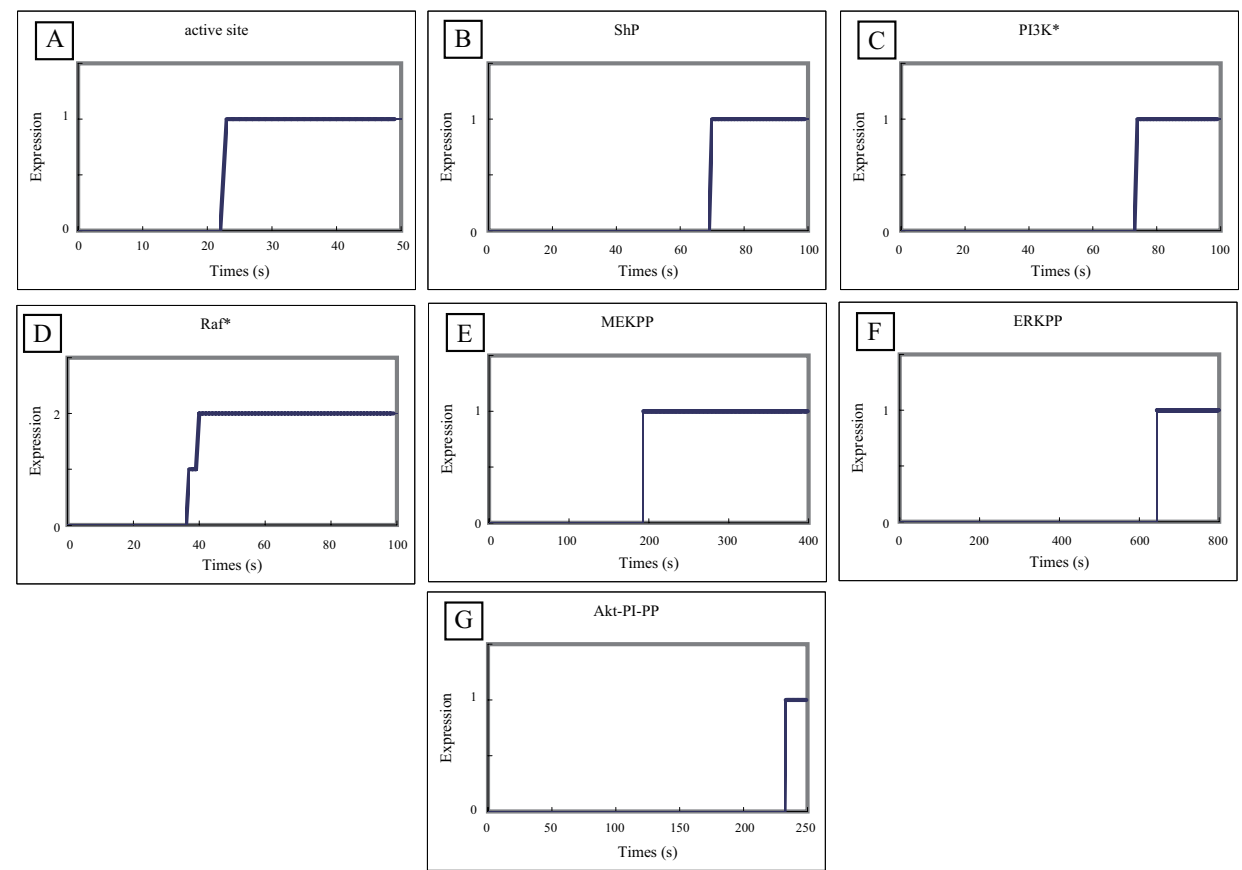

Figure 9: Simulation results of timed Petri net model based on experimental data. (A) Phosphorylation of the ErbB4 receptor (RP), (B) phosphorylation of Shc (ShP), (C) activation of PI3K (PI3K), (D) activation of Raf-1 (Raf), (E) activation of MEK (MEKPP), (F) activation of ERK (ERKPP), (G) activation of Akt (Akt-PI-PP).

\section{CONCLUSIONS}

In this paper, we proposed a delay time estimation algorithm for Petri net model of signaling pathways based on biological experimental data. We first modeled a discrete Petri nets model of ErbB4 receptor signaling pathway using the modeling method proposed by Li et al. [1], and then we assigned a delay time to each transition by using the proposed estimation algorithm based on the timing data of signal transductions of ErbB4 signaling pathway given in [3]. By conducting the simulation of the delay-time-assigned Petri net model on Cell Illustrator, we have confirmed that token flows in this Petri net model reflect the used timing data.

With a Petri net model of a signaling pathway with delays obtained by the proposed algorithm, we can observe signal flows by the token movements. However, ordinary pathway simulations produce concentration behaviors of pathway elements (e.g. proteins, nucleotides, and other bio-chemical substances), providing more precise and systematic observations for the understanding of signaling pathways. Hence, our next task is to develop a method to convert a (discrete) Petri net using discrete delay times to a hybrid Petri net [7] using continuous speeds. Hybrid Petri net has continuous elements (continuous places and transitions) as well as elements of discrete Petri net. The converted hybrid Petri net can be simulated on Cell Illustrator because this simulation tool employs hybrid Petri net as its basic architecture. This new modeling scheme for signaling pathways leads us to the automatic construction of pathway models for numerical simulations.

\section{ACKNOWLEDGMENTS}

This work was partially supported by Grant-in-Aid for Scientific Research on Priority Areas "Systems Genomics" (17017008) from Ministry of Education, Culture, Sports, Science and Technology of Japan.

\section{REFERENCES}

[1] C. Li, S. Suzuki, Q.W. Ge, M. Nakata, H. Matsuno, and S. Miyano. Structural modeling and analysis of signaling pathways based on Petri nets. Journal of Bioinformatics and Computational Biology, 4(5):1119-1140, 2006.

[2] C. Li, Q.W. Ge, M. Nakata, H. Matsuno, and S. Miyano. Modeling and simulation of signal transductions in an apoptosis pathway by using timed Petri nets. Journal of Biosciences, 32(1):113-127, 2007.

[3] M. Hatakeyama, S. Kimura, T. Naka, T. Kawasaki, N. Yumoto, M. Ichikawa, J.H. Kim, K. saito, M. Seki, M. Shirouzu, S. Yokoyama, and A. Konagaya. A computational model on the modulation of mitogen-activated protein kinase (MAPK) and Akt pathways in heregulin-induced ErbB signaling. Biochemical Journal, 373(2):451-263, 2003.

[4] J.L. Peterson. Petri net theory and the modeling of systems. Prentice-hall, 1981.

[5] H. Matsuno, C. Li, and S. Miyano. Petri net based descriptions for systematic understandings of biological pathways. IEICE Trans. Fundamentals., E89-A(11):3166-3174, 2006.

[6] G. Koh, H.F.C. Fern, M. Clement, D. Hsu, and P.S. Thiagarajan. A decompositional approach to parameter estimation in pathway modeling: a case study of the Akt and MAPK pathways and their crosstalk. Bioinformatics, 22(14):e271-e280, 2006.

[7] H. Alla, and R. David, Continuous and hybrid petri nets. Journal of Circuits, Systems, and Computers, 8(1):159-188, 1998.

[8] Cell Illustrator. http://www.cellillustrator.com/ 